\title{
Mitigating inter-cluster interference on the uplink for a three-cell clustered cooperative network
}

\author{
Ajay Thampi ${ }^{*}$, Simon Armour ${ }^{1}$, Zhong Fan ${ }^{2}$ and Dritan Kaleshi ${ }^{1}$
}

\begin{abstract}
In this paper, we propose a practical and scalable solution to mitigating interference on the uplink through static clustering and adaptive fractional frequency reuse (CFFR). The focus is on a three-cell clustered network due to its low complexity. Moreover, we have previously shown that a performance comparable to that of global coordination is achievable using a cluster size of three. In this paper, for a clustered planar Wyner network, we derive analytical capacity equations for zero forcing (ZF) and linear minimum mean squared error (LMMSE)-based receivers. The theoretical results show that inter-cluster interference is the major performance bottleneck and that the smallest interference from the neighbouring clusters is sufficient to significantly lower the system performance. We then switch our study to a more realistic network setting and augment our CFFR technique by adopting an entirely distributed architecture and by implementing a location classification algorithm based on logistic regression. We then show through simulations that CFFR performs significantly better than the widely studied dynamic clustering (DC) technique. Since the inter-cluster interference intensity of CFFR is much lower than DC, the per-cell sum rate performance is $1.5 \times$ better, especially at high loads. We also show that the CFFR algorithm is a lot less complex than DC in terms of running time.
\end{abstract}

Keywords: Network MIMO, Clustered network MIMO, Multi-cell processing, MCP, Joint processing, JP, Base station cooperation, BSC, Fractional frequency reuse, FFR, LTE-A, ZF, LMMSE, Clustering, Cooperation

\section{Introduction}

In modern cellular networks (4G: LTE-A and WiMAX), cell sizes are reducing and reuse factors are approaching 1. A major performance bottleneck in such systems is the interference between cell sites [1]. The problem of interference can be overcome by allowing cells in the network to cooperate. In [2], various multi-cell cooperation techniques are highlighted, grouping them into four broad categories. Two of them that are relevant to this paper are interference coordination and MIMO cooperation.

In interference coordination, the base stations (BSs) share the channel state information (CSI) of the home cell as well as the neighbouring interfering cells. The data transmitted to the mobile stations (MSs) is however not shared. A promising interference coordination strategy is

\footnotetext{
*Correspondence: ajay.thampi@gmail.com

${ }^{1}$ Department of Electrical and Electronic Engineering, University of Bristol, Woodland Road, Bristol, UK

Full list of author information is available at the end of the article
}

fractional frequency reuse (FFR). FFR finds its roots in [3], and it has grown in popularity in $4 \mathrm{G}$ systems based on OFDMA [4-8]. In FFR, a reuse factor of 1 is used for MSs in the cell-centre and a greater reuse factor is used for MSs near the cell-edge. This is because cell-edge users are more prone to inter-cell interference than cell-centre users.

In MIMO cooperation, on the other hand, BSs share not just the CSI but also the data that is transmitted to the users in all the cooperating cells. This is made possible by connecting all the BSs with high-capacity backhaul links. Since both the CSI and data are shared between cell sites, interference can be mitigated by either precoding the signals on the downlink so that they can be successfully decoded at the receivers or by jointly decoding the received signals across cell sites on the uplink. In this strategy, interference is not treated as noise and it is instead exploited as useful information. In the literature, MIMO cooperation is sometimes also referred to as network MIMO [9], base station cooperation (BSC) ([10],

\section{Springer Open}

(C) 2016 Thampi et al. Open Access This article is distributed under the terms of the Creative Commons Attribution 4.0 International License (http://creativecommons.org/licenses/by/4.0/), which permits unrestricted use, distribution, and reproduction in any medium, provided you give appropriate credit to the original author(s) and the source, provide a link to the Creative Commons license, and indicate if changes were made. 
chapter 1), joint processing (JP) ([10], chapter 15) under the coordinated multi-point transmission and reception (CoMP) umbrella [11] or multi-cell processing (MCP) $[2,12,13]$. Henceforth, the term used in this paper will be MCP.

The ideal solution to combat interference is to allow all the BSs to coordinate and to perform MCP on a global scale. This is however highly complex as a large amount of data and CSI exchange is required. In this paper, we focus on finding a scalable solution to overcoming interference on the uplink.

\subsection{Related work}

A practical and scalable solution to global MCP is to group a limited number of BSs into clusters and perform MCP locally in each cluster. In [14] and [15], a basic static clustering approach with linear beamforming is proposed and the spectral efficiency is shown to improve linearly with the number of cooperating BSs. Inter-cluster interference is however not explicitly mitigated. A solution using helper clusters is proposed in [16]. In such a system, interference within the cluster is mitigated by using the block diagonalisation [17] linear precoding technique. The neighbouring clusters are then allowed to act as helpers to cancel some of the interference between clusters. Not all neighbouring clusters will help out, and hence, inter-cluster interference is not entirely cancelled. It is shown in [16] that with a sufficiently large cluster size ( $\geq 7 \mathrm{BSs}$ ), the sum rate performance approaches that of global coordination. The approach in [16] is also shown to perform better than static clustering.

Another approach that is widely studied in the literature is dynamic clustering (DC), where clusters of BSs are formed in a dynamic manner depending on the nature of the channel conditions. Due to the distributed nature of MCP, it is shown to exploit macrodiversity and provide performance gains by taking into account changing channel conditions $[18,19]$. The intuitive reason for the performance gains is that since BSs within a cluster change dynamically, no regions within the cell are always prone to interference. Efficient, iterative algorithms are proposed in [18, 20-25], and significant performance gains are observed for a small cluster size $(\leq 4 \mathrm{BSs})$. DC requires a hybrid architecture where the clustering algorithm runs on a central server, and then MCP is performed in each cluster in a distributed manner. We will discuss each of these DC techniques in greater detail in Section 4.1 and pick the best one to compare with our proposed approach.

In [26], we proposed a static clustering and adaptive FFR approach to combat interference. We call such a system clustered FFR or CFFR. In CFFR, like in [14-16], clusters are statically formed and inter-cluster interference is mitigated by allocating different sub-bands for cluster-centre and cluster-edge users. We apply FFR on a cluster scale and use a reuse factor of 1 for cluster-centre users and a greater reuse factor for cluster-edge users. In addition, the bandwidth partitions are determined in an adaptive manner based on the load in each cluster. For instance, if all the users are in the cluster-centre, then they are allowed to use the entire bandwidth so as to maximise system performance. If, on the other hand, there are more users in the cluster-edge, then the focus of the algorithm will be to cancel inter-cluster interference by allocating different sub-bands for edge users in each cluster. Thus, in such a system, we need to be able to classify users as either cluster-centre or cluster-edge. We show that with just a cluster size of 3, CFFR performs significantly better than [16] and in turn [14] and [15]. It is also shown to perform better than applying FFR in each cell [27]. In addition, the sum rate performance is shown to be close to that of global MCP.

There are however some gaps in our study in [26]. Firstly, we limit our study to the downlink using the block diagonalisation precoding technique and assume ideal location classification where users are accurately classified as either cluster-centre or cluster-edge. Secondly, we have not compared the performance of CFFR with the widely studied DC technique. Thirdly, we assume a hybrid architecture in [26] where the bandwidth partitions are determined by a central server after which, precoding is done independently in each cluster. The performance of [26] using an entirely distributed architecture is unknown. These gaps are addressed in this paper.

\subsection{Contributions}

In this paper, we focus on the uplink of a three-cell clustered cooperative network. We limit our study to a cluster size of three because it significantly lowers the overheads of data and CSI exchange and decoding. Moreover, we have shown in [26] that we can achieve a performance comparable to that of global MCP. We consider two linear decoding techniques namely, zero forcing (ZF) and linear minimum mean squared error (LMMSE) to overcome intra-cluster interference.

We first study theoretically the impact of inter-cluster interference on the per-cell sum rate. To make the problem mathematically tractable, we modify the planar Wyner network [28] to model a clustered system. We derive capacity equations for clustered MCP using ZF and LMMSE decoders in terms of intra-cluster and inter-cluster interference intensities. To the best of our knowledge, this has not been studied in the literature. We show that inter-cluster interference is the major performance bottleneck and that a very small interference intensity is sufficient to significantly lower the overall system performance.

We then consider a more realistic CFFR system that adopts an entirely distributed architecture, i.e. bandwidth 
partitioning and MCP are performed in each cluster independently. We also implement a simple location classification algorithm based on logistic regression on a cluster scale. We call such a system CFFR-LR and compare its performance with that of the ideal system that we proposed in [26], which we will call CFFR-ideal. In addition, we will also compare the performance of CFFR-LR and CFFR-ideal with the widely studied DC technique. The performance comparison is done by simulating a more realistic network environment. We show that CFFR-LR and CFFR-ideal lower the inter-cluster interference intensity significantly when compared to DC. This in turn translates into much higher per-cell sum rates, and we use the theoretical study to better understand the differences. We also show that the performance of CFFR-LR is close to that of CFFR-ideal. Finally, we recommend using the simpler ZF decoding technique for CFFR since the SINR performance is significantly better than DC.

The remainder of the paper is structured as follows. In Section 2, we describe the general system model and restrict the scope of our study to a three-cell clustered network. Section 3 then introduces the modified planar Wyner model and we derive analytical equations for the capacities using ZF and LMMSE decoders. Once we have a theoretical handle of the impact of inter-cluster interference on the performance, we study the two interference mitigation techniques namely, DC and CFFR, in great detail in Section 4. Section 5 then compares the performance of the two strategies, in terms of inter-cluster interference intensity, per-cell sum rate, and execution time, in a more realistic network setting. Section 6 then concludes the paper.

\section{System model}

The system consists of $C$ clusters with $B$ BSs in each cluster. Each cell in the network is serviced by one BS. Therefore, the total number of BSs or cells in the system would be $N=B C$. In each cluster, $K$ users are served during each transmission time interval (TTI). Since we are focusing on the uplink (UL), each MS has $N_{\mathrm{T}}$ transmitter antennas and each BS has $N_{R}$ receive antennas. Figure 1 shows a network consisting of $N=21$ cells statically divided into clusters of $B=3$ cells. There are $C=7$ clusters, and they are numbered from 0 to 6 where the home cell is represented by numeral 0 and the surrounding interfering clusters as numerals $1-6$. The cell radius is denoted as $R$. The boundary between the cluster-interior and cluster-edge, which we refer to as the coordination distance, is denoted as $D_{c}$. It was determined in [16] that the optimum $D_{c}=0.35 R$ for the setup considered. A similar setup is also considered in this paper, which is discussed in Section 5.1. Let $l_{k}$ be the length of the data symbol transmitted by user $k$. In this paper, we assume that the length of the transmitted vector by

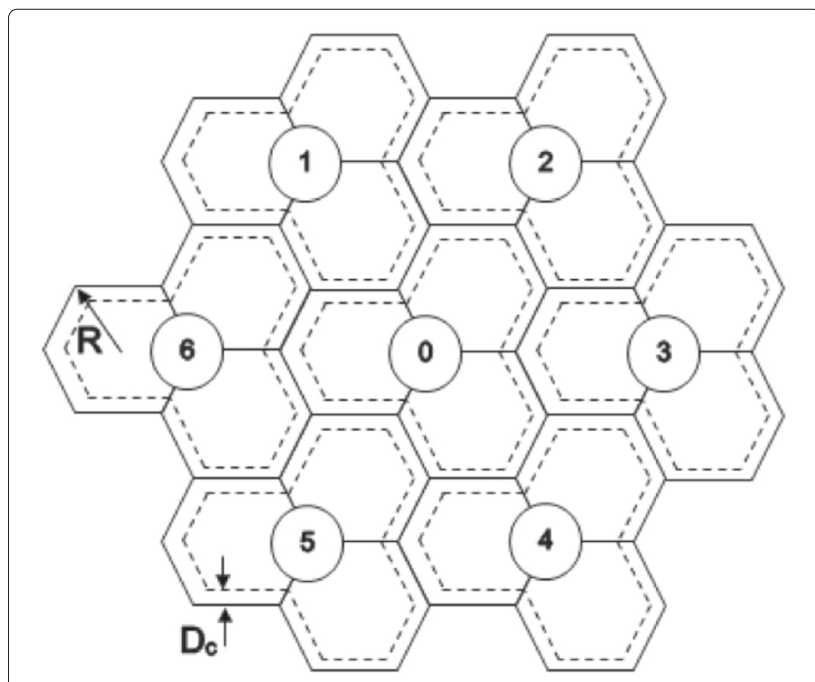

Fig. 1 Static clustering in a multi-cell network

each user is equal to the number of transmit antennas, i.e. $l_{k}=N_{\mathrm{T}}$.

Let $\mathbf{x}_{k}^{(c)}$ be the $N_{\mathrm{T}} \times 1$ transmitted vector by user $k$ in cluster $c$ and $\mathbf{H}_{k}^{(c)}$ be the $B N_{\mathrm{R}} \times N_{T}$ complex channel matrix between user $k$ and all the pooled BSs in cluster $c$. Then, the received $B N_{\mathrm{R}} \times 1$ vector at cluster $c$ from user $k$ is given by,

$$
\begin{aligned}
\mathbf{y}_{k}^{(c)}= & \underbrace{\mathbf{H}_{k}^{(c)} \mathbf{x}_{k}^{(c)}}_{\text {desired signal }}+\underbrace{\sum_{\substack{i=1 \\
i \neq k}}^{K} \mathbf{H}_{i}^{(c)} \mathbf{x}_{i}^{(c)}}_{\text {intra-cluster interference }} \\
& +\underbrace{\sum_{\substack{\hat{c}=0 \\
\hat{i \neq c}}}^{C-1} \sum_{j=1}^{K} \mathbf{H}_{j}^{(\hat{c})} \mathbf{x}_{j}^{(\hat{c})}+\mathbf{n}^{(c)}}_{\text {inter-cluster interference }}
\end{aligned}
$$

where $\mathbf{n}^{(c)} \sim \mathbb{C} \mathcal{N}\left(0, \sigma_{n}^{2} \mathbf{I}_{B N_{\mathrm{R}}}\right)$ is the $B N_{\mathrm{R}} \times 1$ complex additive white Gaussian noise (AWGN) vector at the BSs in cluster $c$.

By combining the transmitted vectors of all users in the network, (1) can be simplified as follows,

$$
\mathbf{y}^{(c)}=\mathbf{H}^{(c)} \mathbf{x}^{(c)}+\mathbf{H}^{(\hat{c})} \mathbf{x}^{(\hat{c})}+\mathbf{n}^{(c)}
$$

where $\mathbf{y}^{(c)}$ is the $B N_{\mathrm{R}} \times 1$ received vector at the pooled BSs from all $K$ users in cluster $c, \mathbf{x}^{(c)}$ is the $K N_{\mathrm{T}} \times 1$ transmitted vector of all $K$ users in cluster $c$ and $\mathbf{H}^{(c)}$ is the $B N_{\mathrm{R}} \times K N_{\mathrm{T}}$ channel matrix between the users and the BSs in cluster $c$. In addition, $\mathbf{x}^{(\hat{\hat{c}})}$ is the $(C-1) K N_{\mathrm{T}} \times 1$ transmitted vector of all users in the neighbouring $C-1$ clusters and $\mathbf{H}^{(\hat{\hat{c}})}$ is the $B N_{\mathrm{R}} \times(C-1) K N_{\mathrm{T}}$ channel between the users in the neighbouring $C-1$ clusters and the BSs in cluster $c$. 
Since we assume that $l_{k}=N_{\mathrm{T}}$, the maximum number of users that can be scheduled in each cluster during each TTI is,

$$
K_{\max } \leq\left\lfloor\frac{B N_{\mathrm{R}}}{N_{\mathrm{T}}}\right\rfloor
$$

\section{Theoretical performance analysis}

In this section, we take a theoretical approach to understanding the impact of inter-cluster interference on the cell-rate performance. where the ZF and LMMSE intracluster interference coordination strategies are adopted on the uplink.

Assuming the BSs have perfect knowledge of the CSI within the cluster, an estimate of the transmitted vector of all $K$ users in cluster $c$ can be obtained for ZF-based MCP as follows,

$$
\hat{\mathbf{x}}_{\mathrm{ZF}}^{(c)}=\left(\mathbf{H}^{(c)^{H}} \mathbf{H}^{(c)}\right)^{-1} \mathbf{H}^{(c)} \mathbf{y}^{(c)}
$$

where $\mathbf{y}^{(c)}$ is given by (2).

For LMMSE-based MCP, the estimate of the transmitted vector is given by,

$$
\hat{\mathbf{x}}_{\mathrm{LMMSE}}^{(c)}=\mathbf{H}^{(c)^{H}}\left(\mathbf{H}^{(c)} \mathbf{H}^{(c)^{H}}+\sigma_{0}^{2} \mathbf{I}_{B N_{\mathrm{R}}}\right)^{-1} \mathbf{y}^{(c)}
$$

where $\sigma_{0}^{2}$ is the inverse of the received SNR at the BSs in cluster $c$ and $\mathbf{I}_{B N_{\mathrm{R}}}$ is the identity matrix of dimension $B N_{R}$. In the following sub-sections, we derive closed-form capacity equations for ZF and LMMSE for a modified planar Wyner network.

\subsection{Modified planar Wyner model}

We modify the widely studied planar Wyner network $[2,28-32]$ to a three-cell clustered cooperative network. In [28], a two-dimensional hexagonal array of cells is considered, as shown in Fig. 1. This hexagonal array is represented as a checkerboard lattice, and to make it more tractable, it is converted to a rectangular array. In the planar Wyner model, the signal transmitted by a user in the home cell does not experience any attenuation. The signals from neighbouring cells interfere with the home user and the signals are attenuated by a factor of $\alpha$, where $\alpha \in[0,1]$. We modify this rectangular array lattice representation to factor in inter-cluster interference as well. Figure 2 shows the lattice representation for a network with a cluster size of three, and it is obtained by rotating the hexagonal lattice by $45^{\circ}$ and scaling by a factor of $\frac{1}{\sqrt{2}}$ [28]. Interference from only the first-tier neighbouring clusters is considered. This intra-cluster interference intensity is denoted as $\alpha$, as in [28]. We introduce a new parameter for inter-cluster interference and denote it as $\beta$, where $\beta \in[0,1]$. Since the interference intensities are scalar and deterministic parameters, the scaling of the lattice does not affect the interference pattern.

Without a loss of generality, the following simplifying assumptions are made in this section. An intra-cell TDMA transmission strategy is considered where only a single user per cell is transmitting at a given time instance. Thus, the number of active users in a three-cell clustered network is $K=3$. The additive white Gaussian noise (AWGN) channel model is assumed with 0 mean and variance $\sigma_{n}^{2}$. All the BSs have perfect knowledge of the CSI as well as each other's codebooks. The BSs within a cluster are connected using an ideal backhaul with infinite capacity. The BSs are perfectly synchronised to ensure that all signals are received at the same time. BPSK modulation is assumed and the power with which the users transmit the antipodal signals are the same, i.e. no power control is performed across cell sites. Assuming that the received amplitude of each user's signal is $A$, then the received power of each user is $A^{2}$. Since a cluster size of $B=3$ is assumed, there are nine interfering cells from the first-tier neighbouring clusters as can be seen in Fig. 2. Finally, the MSs and BSs are equipped with single, omnidirectional antennas, i.e. $N_{\mathrm{T}}=N_{\mathrm{R}}=1$.

For the three-cell clustered network, the channel matrix between the users and the BSs in cluster $c$ is given by,

$$
\mathbf{H}^{(c)}=\left[\begin{array}{ccc}
1 & \alpha & \alpha \\
\alpha & 1 & \alpha \\
\alpha & \alpha & 1
\end{array}\right]
$$

The channel matrix between the users in the neighbouring clusters and the BSs in cluster $c$ is given by,

$$
\mathbf{H}^{(\hat{c})}=\left[\begin{array}{lllllllll}
\beta & \beta & \beta & \beta & 0 & 0 & 0 & 0 & 0 \\
0 & 0 & \beta & \beta & \beta & \beta & 0 & 0 & 0 \\
\beta & 0 & 0 & 0 & 0 & 0 & \beta & \beta & \beta
\end{array}\right]
$$

Now, (2) can be rewritten as,

$$
\begin{aligned}
\mathbf{y}^{(c)} & =\mathbf{H}^{(c)} \mathbf{x}^{(c)}+\mathbf{y}^{(\hat{\hat{c}})}+\mathbf{n}^{(c)} \\
& =\mathbf{H}^{(c)} \mathbf{x}^{(c)}+\tilde{\mathbf{n}}^{(c)}
\end{aligned}
$$

where $\mathbf{y}^{(\hat{\hat{c}})}=\mathbf{H}^{(\hat{\hat{c}})} \mathbf{x}^{(\hat{\hat{c}})}$ and $\tilde{\mathbf{n}}^{(c)}$ combines the interference from the neighbouring clusters and the noise at the receivers of the home cluster. The covariance matrix of $\tilde{\mathbf{n}}^{(c)}$ is then given by,

$$
\begin{aligned}
\mathbb{E}\left[\tilde{\mathbf{n}}^{(c)} \tilde{\mathbf{n}}^{(c)^{T}}\right]= & \mathbb{E}\left[\left(\mathbf{y}^{(\hat{\hat{c}})}+\mathbf{n}^{(c)}\right)\left(\mathbf{y}^{(\hat{\hat{c}})}+\mathbf{n}^{(c)}\right)^{T}\right] \\
= & \mathbb{E}\left[\mathbf{y}^{(\hat{\hat{c}})} \mathbf{y}^{(\hat{\hat{c}})^{T}}\right]+\mathbb{E}\left[\mathbf{y}^{(\hat{\hat{c}})} \mathbf{n}^{\left.(c)^{T}\right]}\right. \\
& \left.+\mathbb{E}\left[\mathbf{n}^{(c)} \mathbf{y}^{(\hat{\hat{c}}}\right)^{T}\right]+\mathbb{E}\left[\mathbf{n}^{(c)} \mathbf{n}^{(c)^{T}}\right] \\
= & \left(4 \beta^{2} A^{2}+\sigma_{n}^{2}\right) \mathbf{I}_{3}
\end{aligned}
$$



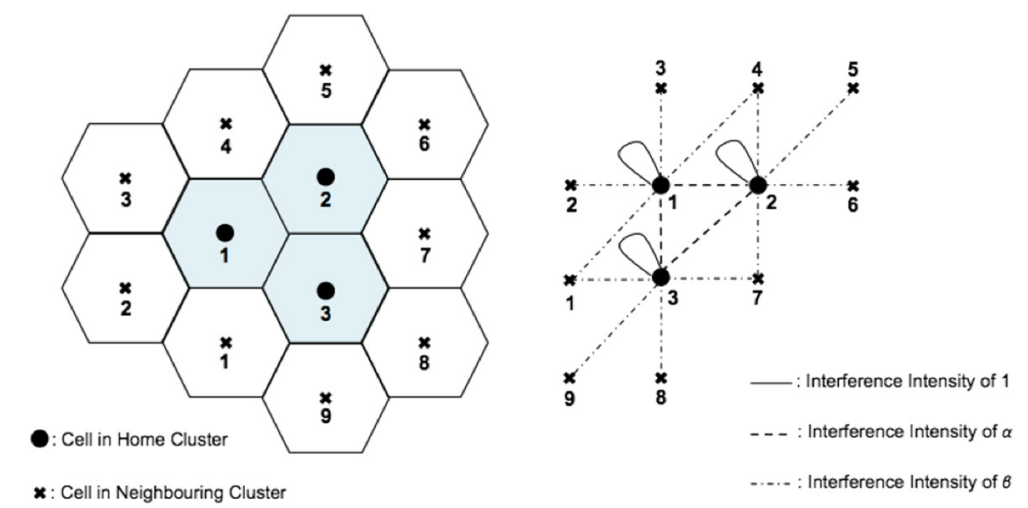

Fig. 2 Three-cell clustered planar Wyner network

In (9), the first term equates to the received power of the users in the neighbouring clusters at each cell in cluster $c$, which is $4 \beta^{2} A^{2} \mathbf{I}_{3}$. The second and third terms equate to 0 since the signals transmitted by the users and the noise are uncorrelated. Since the AWGN channel model with 0 mean and variance $\sigma_{n}^{2}$ is considered, the final term in (9) equates to $\sigma_{n}^{2} \mathbf{I}_{3}$. Combining all these terms gives us (10).

\subsection{Capacity of ZF}

The estimate of the transmitted vector using ZF receivers is given by,

$$
\begin{aligned}
\hat{\mathbf{x}}^{(c)} & =\left(\mathbf{H}^{(c)^{T}} \mathbf{H}^{(c)}\right)^{-1} \mathbf{H}^{(c)} \mathbf{y}^{(c)} \\
& =\mathbf{H}^{(c)^{-2}} \mathbf{H}^{(c)} \mathbf{y}^{(c)} \\
& =\mathbf{H}^{(c)^{-1}} \mathbf{y}^{(c)} \\
& =\mathbf{x}^{(c)}+\mathbf{H}^{(c)} \tilde{\mathbf{n}}^{(c)}
\end{aligned}
$$

Since the channel matrix is real in the modified Wyner network, we take the transpose of $\mathbf{H}^{(c)}$ in (11) rather than the Hermitian given by (4). Step (12) is obtained since $\mathbf{H}^{(c)}$, given by (6) is symmetric. Finally, (14) is obtained by substituting (8) in (13).

Theorem 1. The per-cell capacity of a three-cell clustered network using ZF is given by,

$$
C_{Z F}=\frac{1}{2} \log _{2}\left[1+\frac{A^{2}(1-\alpha)^{2}(1+2 \alpha)^{2}}{\left(4 \beta^{2} A^{2}+\sigma_{n}^{2}\right)\left(2 \alpha^{2}+(1+\alpha)^{2}\right)}\right]
$$

Proof. From (14), the received power of all users in cluster $c$ is given by $A^{2} \mathbf{I}_{3}$, and the noise power is given by the covariance matrix of the background noise $\left(\mathbf{H}^{(c)}\right)^{-1} \tilde{\mathbf{n}}^{(c)}$, given by

$$
\begin{aligned}
\mathbb{E}[ & \left.\left(\mathbf{H}^{(c)^{-1}} \tilde{\mathbf{n}}^{(c)}\right)\left(\mathbf{H}^{(c)^{-1}} \tilde{\mathbf{n}}^{(c)}\right)^{T}\right] \\
& =\mathbb{E}\left[\mathbf{H}^{(c)^{-1}} \tilde{\mathbf{n}}^{(c)} \tilde{\mathbf{n}}^{(c)^{T}} \mathbf{H}^{(c)^{-T}}\right] \\
& =\mathbf{H}^{(c)^{-1}} \mathbb{E}\left[\tilde{\mathbf{n}}^{(c)} \tilde{\mathbf{n}}^{(c)^{T}}\right] \mathbf{H}^{(c)^{-T}} \\
& =\mathbf{H}^{(c)^{-1}}\left(4 \beta^{2} A^{2}+\sigma_{n}^{2}\right) \mathbf{I}_{3} \mathbf{H}^{(c)^{-1}} \\
& =\left(4 \beta^{2} A^{2}+\sigma_{n}^{2}\right) \mathbf{H}^{(c)^{-2}}
\end{aligned}
$$

Since $\mathbf{H}^{(c)}$ is deterministic in the Wyner network, we get (17) from (16). Step (18) is then obtained by substituting (10) in (17). The achievable capacity at cell $i$ is then,

$$
\mathrm{C}_{\mathrm{ZF}}^{(i)}=\frac{1}{2} \log _{2}\left[1+\frac{A^{2}}{\left(4 \beta^{2} A^{2}+\sigma_{n}^{2}\right) \sum_{j=1}^{3}\left(\left(\mathbf{H}^{(c)}\right)_{i j}^{-1}\right)^{2}}\right]
$$

Using the Gauss-Jordan method, we can obtain

$$
\mathbf{H}^{(c)^{-1}}=\frac{1}{(1-\alpha)(1+2 \alpha)}\left[\begin{array}{ccc}
1+\alpha & -\alpha & -\alpha \\
-\alpha & 1+\alpha & -\alpha \\
-\alpha & -\alpha & 1+\alpha
\end{array}\right]
$$

Thus,

$$
\sum_{j=1}^{3}\left(\left(\mathbf{H}^{(c)}\right)_{i j}^{-1}\right)^{2}=\frac{(1+\alpha)^{2}+2 \alpha^{2}}{(1-\alpha)^{2}(1+2 \alpha)^{2}} ; \forall i
$$

The theorem is proved by substituting (22) in (20).

It can be observed from (15) that there is a singularity at $\alpha=1$ which can be overcome by considering fading and multi-user scheduling as shown in [31]. 


\subsection{Capacity of LMMSE}

For the modified Wyner network, the estimate of the transmitted vector using LMMSE receivers is given by,

$$
\hat{\mathbf{x}}_{\text {LMMSE }}^{(c)}=\mathbf{H}^{(c)^{T}}\left(\mathbf{H}^{(c)} \mathbf{H}^{(c)^{T}}+\sigma_{0}^{2} \mathbf{I}_{3}\right)^{-1} \mathbf{y}^{(c)}
$$

In (23), $\sigma_{0}^{2}$ is the inverse of the received SNR at cluster $c$, given by

$$
\sigma_{0}^{2}=\frac{4 \beta^{2} A^{2}+\sigma_{n}^{2}}{A^{2}}
$$

where $4 \beta^{2} A^{2}$ is the interference power from the users in the neighbouring clusters.

In [30], the capacity of an MCP system using LMMSE receivers is derived for a Wyner model where the cells are arranged in a one-dimensional linear array. In this linear model, a finite number of cells $N$ cooperate and only two neighbouring cells interfere with the home cell. The $N \times N$ channel matrix for such a system is given by,

$$
\mathbf{H}=\left[\begin{array}{ccccccc}
1 & \alpha & 0 & 0 & 0 & \cdots & 0 \\
\alpha & 1 & \alpha & 0 & 0 & \cdots & 0 \\
0 & \alpha & 1 & \alpha & 0 & \cdots & 0 \\
\vdots & \ddots & \ddots & \ddots & \ddots & \ddots & \vdots \\
0 & \cdots & \cdots & 0 & \alpha & 1 & \alpha \\
0 & \cdots & \cdots & \cdots & 0 & \alpha & 1
\end{array}\right]
$$

The achievable capacity of cell $i$ is then shown in [30] to be,

$$
\begin{aligned}
C_{\mathrm{LMMSE}}^{(i)}= & -\frac{1}{2} \log _{2}\left[1-\frac{1}{N+1} \sum_{k=1}^{N} \frac{\mu_{k}^{2}}{\mu_{k}^{2}+\sigma_{0}^{2}}\right. \\
& \left.\times\left(1-\cos \left(\frac{2 i k \pi}{N+1}\right)\right)\right]
\end{aligned}
$$

where $\mu_{k} \mathrm{~s}$ are the eigenvalues of $\mathbf{H}$ and $\sigma_{0}^{2}=\frac{\sigma_{n}^{2}}{A^{2}}$. This equation is however not applicable in a clustered MCP system since the channel matrix is different and also since the effects of inter-cluster interference are not considered. We have to therefore re-derive the capacity for a three-cell clustered network.

Following the derivation as in [30] and [33], the LMMSE estimator for user $i$ in cluster $c$ can be shown to be,

$$
\begin{aligned}
\hat{x}_{i}^{(c)} & =\mathbf{h}_{i}^{(c)^{T}}\left(\mathbf{H}^{(c)} \mathbf{H}^{(c)^{T}}+\sigma_{0}^{2} \mathbf{I}_{3}\right)^{-1} \mathbf{y}^{(c)} \\
& =\mathbf{h}_{i}^{(c)^{T}}\left(\mathbf{H}^{(c)^{2}}+\sigma_{0}^{2} \mathbf{I}_{3}\right)^{-1} \mathbf{y}^{(c)}
\end{aligned}
$$

Since $\mathbf{H}^{(c)}$ is symmetric, we get (28) from (27). Let the error of estimation for user $i$ be $W_{i}=x_{i}^{(c)}-\hat{x}_{i}^{(c)}$. The mean-squared error (MSE) of estimation is then given by,

$$
\bar{W}_{i}^{2}=A^{2}\left(1-\mathbf{h}_{i}^{(c)^{T}}\left(\mathbf{H}^{(c)^{2}}+\sigma_{0}^{2} \mathbf{I}_{3}\right)^{-1} \mathbf{h}_{i}^{(c)}\right)
$$

The eigendecomposition of the square matrix, $\mathbf{H}^{(c)^{2}}+$ $\sigma_{0}^{2} \mathbf{I}_{3}$, can be expressed as

$$
\mathbf{H}^{(c)^{2}}+\sigma_{0}^{2} \mathbf{I}_{3}=\mathbf{Q}^{(c)} \Lambda^{(c)}\left(\mathbf{Q}^{(c)}\right)^{T}
$$

where $\mathbf{Q}^{(c)}=\left[\mathbf{q}_{1}^{(c)} \mathbf{q}_{2}^{(c)} \mathbf{q}_{3}^{(c)}\right]$ is the $3 \times 3$ orthogonal matrix consisting of the orthonormal eigenvectors of $\mathbf{H}^{(c)^{2}}+\sigma_{0}^{2} \mathbf{I}_{3}$ and $\Lambda^{(c)}=\operatorname{diag}\left\{\lambda_{1}^{(c)}, \lambda_{2}^{(c)}, \lambda_{3}^{(c)}\right\}$ is the diagonal matrix containing the eigenvalues of $\mathbf{H}^{(c)^{2}}+\sigma_{0}^{2} \mathbf{I}_{3}$.

The eigenvalues $\lambda_{k}^{(c)}$ are related to the eigenvalues of $\mathbf{H}^{(c)}$, denoted as $\mu_{k}^{(c)}$, as follows

$$
\lambda_{k}^{(c)}=\mu_{k}^{(c)^{2}}+\sigma_{0}^{2} ; k=1,2,3
$$

On the other hand, the eigenvectors of $\mathbf{H}^{(c)^{2}}+\sigma_{0}^{2} \mathbf{I}_{3}$, denoted as $\mathbf{q}_{k}^{(c)}$, are also the eigenvectors of $\mathbf{H}^{(c)}$.

The eigenvalues and eigenvectors of $\mathbf{H}^{(c)}$, given by (6), exhibit the following properties:

1. The eigenvalues, $\mu_{k}^{(c)}$ are:

$$
\begin{aligned}
& \mu_{k}^{(c)}=1+2 \alpha \cos \left(\frac{2(k-1) \pi}{3}\right) ; k=1,2,3 \\
& \text { i.e. } \mu_{1}^{(c)}=(1+2 \alpha) \text { and } \mu_{2}^{(c)}=\mu_{3}^{(c)}=(1-\alpha) .
\end{aligned}
$$

2. The eigenvectors, $\mathbf{q}_{k}^{(c)}$ are independent and orthonormal. i.e.

$$
\begin{aligned}
& \sum_{j=1}^{3} q_{k j}^{(c)}=0 ; k=1,2,3 \\
& \sum_{k=1}^{3} q_{k j}^{(c)^{2}}=1 ; j=1,2,3
\end{aligned}
$$

3. It can be shown that

$$
q_{1 j}^{(c)}=\left(\frac{1}{3}\right)^{\frac{1}{2}} ; j=1,2,3 .
$$

Theorem 2. The per-cell capacity of a three-cell clustered network using LMMSE is given by,

$$
\begin{aligned}
C_{\text {LMMSE }}= & -\frac{1}{2} \log _{2}\left[1-\frac{1}{3}\left(\frac{(1+2 \alpha)^{2}}{(1+2 \alpha)^{2}+\sigma_{0}^{2}}\right.\right. \\
& \left.\left.+\frac{2(1-\alpha)^{2}}{(1-\alpha)^{2}+\sigma_{0}^{2}}\right)\right]
\end{aligned}
$$

where $\sigma_{0}^{2}=\frac{4 \beta^{2} A^{2}+\sigma_{n}^{2}}{A^{2}}$. 
Proof. Using the eigendecomposition in (30), we can obtain the following,

$$
\begin{aligned}
\mathbf{h}_{i}^{(c)^{T}} & \left(\mathbf{H}^{(c)^{2}}+\sigma_{0}^{2} \mathbf{I}_{3}\right)^{-1} \mathbf{h}_{i}^{(c)} \\
= & \sum_{k=1}^{3} \frac{1}{\lambda_{k}^{(c)}}\left(\alpha q_{k 1}^{(c)}+q_{k 2}^{(c)}+\alpha q_{k 3}^{(c)}\right)^{2} \\
= & \frac{(1+2 \alpha)^{2}}{3 \lambda_{1}^{(c)}}+\frac{1}{\lambda_{2}^{(c)}}\left[\left(\alpha q_{21}^{(c)}+q_{22}^{(c)}+\alpha q_{23}^{(c)}\right)^{2}\right. \\
& \left.+\left(\alpha q_{31}^{(c)}+q_{32}^{(c)}+\alpha q_{33}^{(c)}\right)^{2}\right] \\
= & \frac{(1+2 \alpha)^{2}}{3 \lambda_{1}^{(c)}}+\frac{(1-\alpha)^{2}}{\lambda_{2}^{(c)}}\left[q_{22}^{(c)}+q_{32}^{(c)}\right] \\
= & \frac{(1+2 \alpha)^{2}}{3 \lambda_{1}^{(c)}}+\frac{(1-\alpha)^{2}}{\lambda_{2}^{(c)}}\left[1-q_{12}^{(c)}\right] \\
= & \frac{(1+2 \alpha)^{2}}{3 \lambda_{1}^{(c)}}+\frac{2(1-\alpha)^{2}}{3 \lambda_{2}^{(c)}} \\
= & \frac{1}{3}\left[\frac{(1+2 \alpha)^{2}}{(1+2 \alpha)^{2}+\sigma_{0}^{2}}+\frac{2(1-\alpha)^{2}}{(1-\alpha)^{2}+\sigma_{0}^{2}}\right]
\end{aligned}
$$

Step (38) can be obtained by substituting (32) and (35) in (37). Steps (39), (40), (41) and (42) can be obtained from (33), (34), (35) and (32), respectively. By substituting (42) in (29), we get

$\bar{W}_{i}^{2}=A^{2}\left[1-\frac{1}{3}\left(\frac{(1+2 \alpha)^{2}}{(1+2 \alpha)^{2}+\sigma_{0}^{2}}+\frac{2(1-\alpha)^{2}}{(1-\alpha)^{2}+\sigma_{0}^{2}}\right)\right]$

The per-cell capacity can then be obtained by computing the mutual information of $x_{i}^{(c)}$ and $\hat{x}_{i}^{(c)}$, denoted as $I\left(x_{i}^{(c)} ; \hat{x}_{i}^{(c)}\right)$. The MSE of the estimation is given by (43). It is shown in [28] that $I\left(x_{i}^{(c)} ; \hat{x}_{i}^{(c)}\right)=H\left(x_{i}^{(c)}\right)-H\left(W_{i}\right)$. Since all the users are transmitting at the highest power, $A^{2}$, the per-cell capacity is given by,

$$
\begin{aligned}
C_{\mathrm{LMMSE}}^{(i)}= & \frac{1}{2} \log _{2} \frac{A^{2}}{\bar{W}_{i}^{2}} \\
= & -\frac{1}{2} \log _{2}\left[1-\frac{1}{3}\left(\frac{(1+2 \alpha)^{2}}{(1+2 \alpha)^{2}+\sigma_{0}^{2}}\right.\right. \\
& \left.\left.+\frac{2(1-\alpha)^{2}}{(1-\alpha)^{2}+\sigma_{0}^{2}}\right)\right] ; \forall i
\end{aligned}
$$

Equation (45) can be obtained by substituting (43) in (44), thus proving the theorem.

\subsection{Performance analysis}

We plot the capacities of ZF and LMMSE for various interference intensities in Fig. 3 using the equations derived earlier. Two intra-cluster and four different inter-cluster interference intensities are considered, i.e. $\alpha \in\{0.5,0.8\}$ and $\beta \in\{0,0.005,0.1,0.2\}$. It must also be noted that these results have been validated using Monte Carlo simulations.

It can be observed that for all inter-cluster interference intensities, the performances of ZF and LMMSE are better for a lower intra-cluster interference intensity. For instance, for $\beta=0$ and a very high SNR of $60 \mathrm{~dB}$, the performance of ZF and LMMSE for $\alpha=0.5$ is $12.5 \%$ higher than that of $\alpha=0.8$. The same is true even for low to medium SNRs. Also, the gap between ZF and LMMSE for a given $\alpha$ is negligible for $\beta=0$ and $\beta=0.005$. It can be observed that for a small increase in the intensity of inter-cluster interference, the performance of ZF and LMMSE deteriorate significantly only at very high SNRs. For instance, when $\beta$ increases from 0 to 0.005 , the performance of ZF and LMMSE deteriorate by $50 \%$ at $\mathrm{SNR}=60 \mathrm{~dB}$. When $\beta$ increases further from 0.005 to 0.1 , the performance degradation can be observed even at medium SNRs. For instance, when the SNR is $20 \mathrm{~dB}$ the performance degrades by about $72 \%$. It can also be observed that at lower SNRs and higher inter-cluster interference intensities, LMMSE decoders perform better than ZF. We can conclude through this theoretical study that even the slightest inter-cluster interference can seriously deteriorate the performance of a three-cell clustered network.

\section{Inter-cluster interference coordination strategies}

In this section, we discuss two inter-cluster interference coordination strategies: the widely studied dynamic clustering strategy and our proposed strategy of static clustering with adaptive FFR.

\subsection{Dynamic clustering}

As discussed in Section 1.1, dynamic clustering (DC) involves forming clusters of BSs in a dynamic manner based on changing channel conditions. Given a system where $C$ clusters need to be formed with $B$ BSs within a cluster, there are $\frac{N !}{(B !)^{C} C !}$ different cluster realisations [25] where $N=B C$ is the total number of BSs in the network. An exhaustive search through all these cluster realisations for an optimum solution is therefore highly complex. Various efficient algorithms have been proposed in the literature for DC. We will now discuss each of them and will pick the best algorithm to be considered in this paper. 


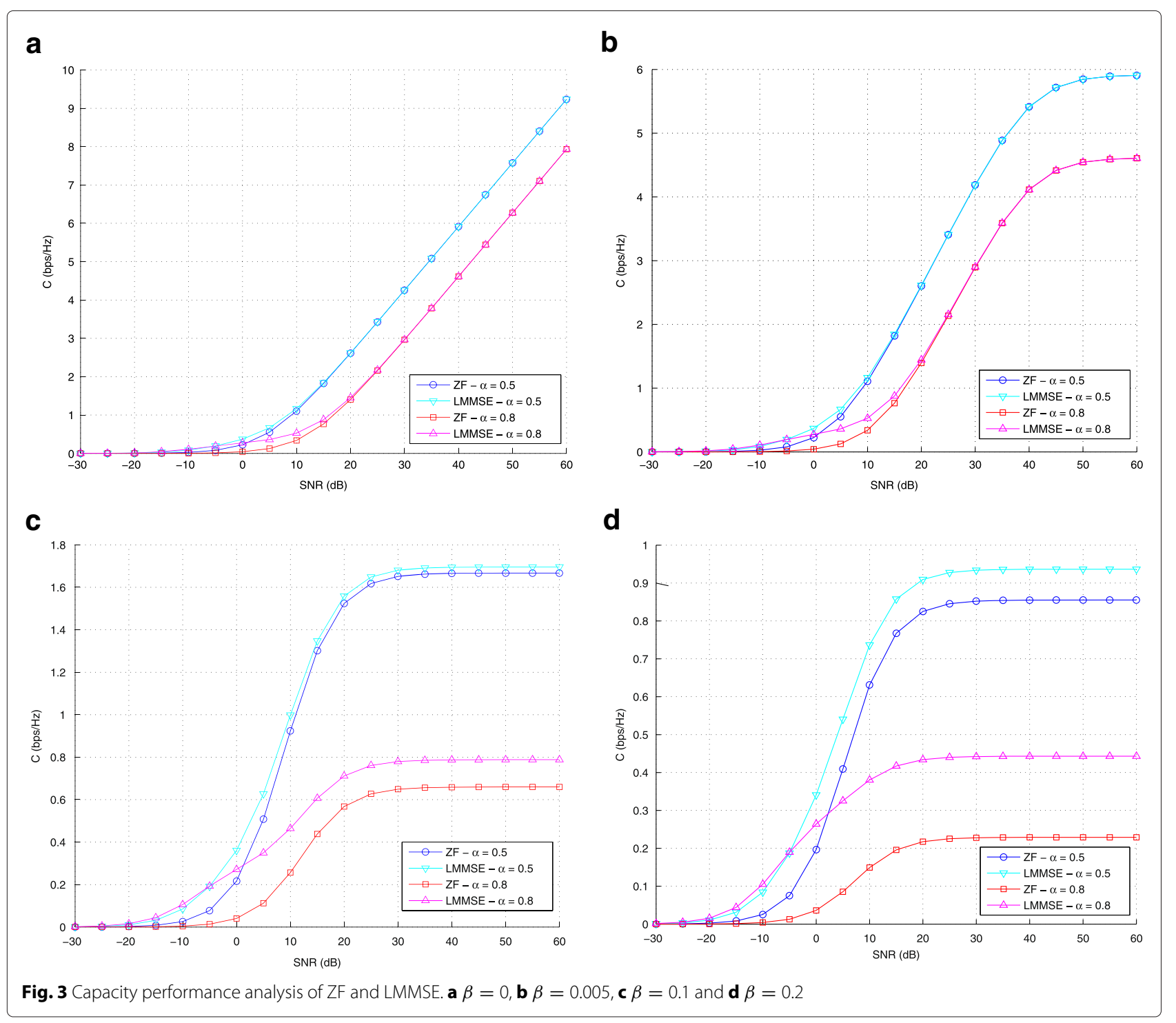

In [18], each $\mathrm{BS}$ in the network collects the instantaneous CSI from all its users and performs scheduling in a decentralised manner. The CSI from all the BSs are then collected by a central server where a greedy clustering algorithm is run. The algorithm picks a random cell and chooses BSs that maximise the joint capacity to form a cluster. This is done in an iterative manner until all the clusters are formed. It must be noted that the cluster size is fixed and remains constant throughout the algorithm. The system in [18] assumes MSs equipped with a single antenna. The algorithm is extended to multi-antenna MSs in [20]. It has also been shown in [21] that CSI from a limited number of BSs is sufficient to guarantee performance.

In [22] and [23], the network is modelled as a graph where the vertices represent the BSs and any two vertices are connected by an edge if the interference between the corresponding BSs is above a certain threshold. Each edge is then assigned a utility value which could be either the average increase in achievable rate or the increase in interference if the two vertices form a cluster. Once the utility values are assigned to all edges, the algorithm iteratively forms clusters so as to maximise the overall utility. The basic idea is however similar to that of [18] in the sense that the algorithm is iterative and maximises the overall sum rate. In addition, the architecture considered is centralised, the size of the cluster remains constant and instantaneous CSI is used to determine the utility values.

The DC algorithm proposed in [18] is improved on in [24] by allowing the cluster size to change dynamically in each iteration. The algorithm runs on a central server and cells that cause the highest amount of interference to each other form a cluster so as to benefit from MCP. The maximum cluster size considered in [24] 
was four, and the algorithm was shown to perform only slightly better than [18] at medium and high SNRs. If the cluster size was larger, then much higher performance gains would have been observed. This would however come at the cost of complexity for clustering as well as MCP.

Finally, [25] proposes a distributed architecture for DC where clustering is performed by each BS in a decentralised manner. Each BS maintains a candidate list of clusters that it would like to join based on a preference function. In [25], the preference function is the average increase in system capacity if the BSs were to form a cluster. As in [18], the size of the cluster remains constant throughout the clustering process. Invitations are then sent to the candidate BSs and each BS maintains an invitation list. Two BSs form a cluster if the pair of them maximise the preference function. A list of BSs that have not yet formed a cluster is also maintained and is expressed as $\epsilon$ in [25]. The iterative clustering algorithm is shown to terminate after at most $C$ iterations, where $C$ is the total number of clusters. The complexity of the decentralised approach is also shown to scale linearly with the network size $N=B C$. Although [25] claims to propose a decentralised framework, it is not entirely so as a central unit is required to maintain the list $\epsilon$. In addition, the overall system performance in terms of sum rate is not better than [18].

Thus, in this paper, the DC algorithm in [18] is used to compare with our proposed algorithm. A hybrid architecture as shown in Fig. 4 is used where clustering is done by a central server, called the CPU. Each BS sends the instantaneous CSI from all its users to the CPU. The iterative greedy algorithm is run on the CPU and the decision is then fed-back to each BS. The BSs that form a cluster then work in a distributed manner to combat intra-cluster interference. This process is repeated on a per-frame or per-slot time scale.

\subsection{Static clustering with adaptive FFR}

The proposed static clustering with adaptive FFR scheme, referred to as CFFR, is shown in Fig. 5. For simplicity, only the home cluster and two neighbouring clusters are considered but the figure can very easily be extended to all 6 neighbouring clusters. Let $W$ be the total available bandwidth. In DC, all users scheduled in the cluster are allowed to use the entire bandwidth. In our CFFR strategy, the bandwidth is divided into cluster-centre $\left(W_{c}\right)$ and cluster-edge bands $\left(W_{e}\right)$. No two neighbouring clusters will share the band for cluster-edge users.

The FFR scheme is adaptive where the bandwidth partitions are made dependent on the load in each cluster. Let $M_{c}^{(i)}$ and $M^{(i)}$ be the number of cluster-centre MSs and the total number of MSs respectively in cluster $i$. Then, the bandwidth allocation for the centre users in cluster $i$ is given by,

$$
W_{c}^{(i)}=\left\lceil\left(\frac{M_{c}^{(i)}}{M^{(i)}}\right) W\right\rceil
$$

Since the focus is on a cluster size of three, the bandwidth allocated for the edge users in cluster $i$ is given by,

$$
W_{e}^{(i)}=\left\lfloor\left(\frac{W-W_{c}^{(i)}}{3}\right)\right\rfloor
$$

We first proposed this CFFR scheme in [26]. In [26], a hybrid architecture is considered where the bandwidth partitioning is done by a central server after which MCP is done in a decentralised manner. This is to ensure that all the clusters share the same FFR scheme. The clusters share its computed bandwidth partitions with the central server and the final partitions; $W_{e}$ and $W_{c}$ are determined such that the largest partition is chosen for the clusteredge users and the corresponding partition for those in the cluster-centre. This is to ensure that the performance for the cluster-edge users is improved. In this paper, we adopt an entirely distributed architecture where the bandwidth partitions and MCP are performed by each cluster in a decentralised manner. This architecture is shown in Fig. 6. Each cluster also performs intra-cluster interference mitigation independently through ZF and LMMSE decoding.

In addition, the location of the users must be classified as either cluster-centre or edge so as to determine the bandwidth partitions and also for scheduling. In [26], we assume an ideal location classifier with $100 \%$ accuracy. We refer to such a system as CFFR-Ideal. In this paper, however, we consider a machine-learning approach of logistic regression for location classification. We extend an algorithm that we proposed in [34] for a single cell to a cluster scale. We call such a system CFFR-LR. In [34], we have shown that using two features, namely, received power and SINR from the MS, the classification accuracy is much better than using a single feature such as SINR [6, 35-37]. We have also shown that the optimum hypothesis is a logistic/sigmoid function, given by (48), based on an affine model. In (48), $z^{(k)}$ is given by (49), where $w_{1}^{(k)}$ is the received power and $w_{2}^{(k)}$ is the SINR reported by user $k$ and $\theta_{0}, \theta_{1}$ and $\theta_{2}$ are the optimum parameters determined through the training process. More details on this can be found in [34].

$$
\begin{aligned}
& f\left(z^{(k)}\right)=\frac{1}{1+\exp \left(-z^{(k)}\right)} \\
& z^{(k)}=\theta_{0}+\theta_{1} w_{1}^{(k)}+\theta_{2} w_{2}^{(k)}
\end{aligned}
$$

In this paper, we consider the user locations on a cluster scale and this would in-turn impact the training phase in 


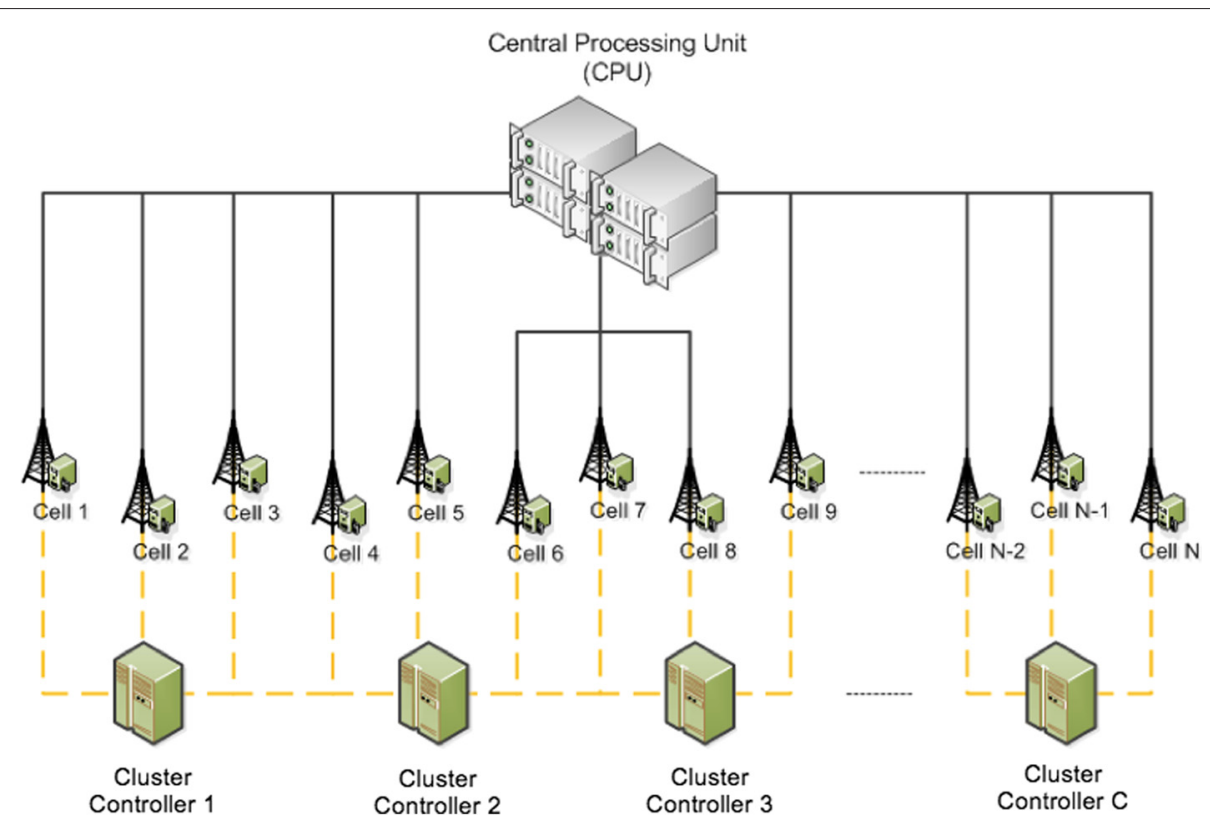

Fig. 4 DC hybrid architecture

the algorithm. The performance of LR on a cluster scale as well as the performance of CFFR with sub-optimum location classification are unknown, and these gaps are addressed in this paper.

\section{Realistic network performance analysis}

In this section, we perform a simulation-based study of the performance of DC, CFFR-LR and CFFR-Ideal in a realistic network setup. In Section 3, we derived capacity equations for ZF and LMMSE for a three-cell clustered network in terms of intra-cluster and inter-cluster interference intensities, $\alpha$ and $\beta$, respectively. The model that we considered was however simple and deterministic, but it helped us understand that even a small $\beta$ can reduce the cell-rate performance significantly. We will now switch our study to a more realistic network in order to determine which is the best inter-cluster interference coordination strategy in terms of lowering $\beta$.

\subsection{Simulation setup}

We consider a network of 21 cells divided up into clusters of size 3. Each MS is equipped with $N_{\mathrm{T}}=2$ antennas and

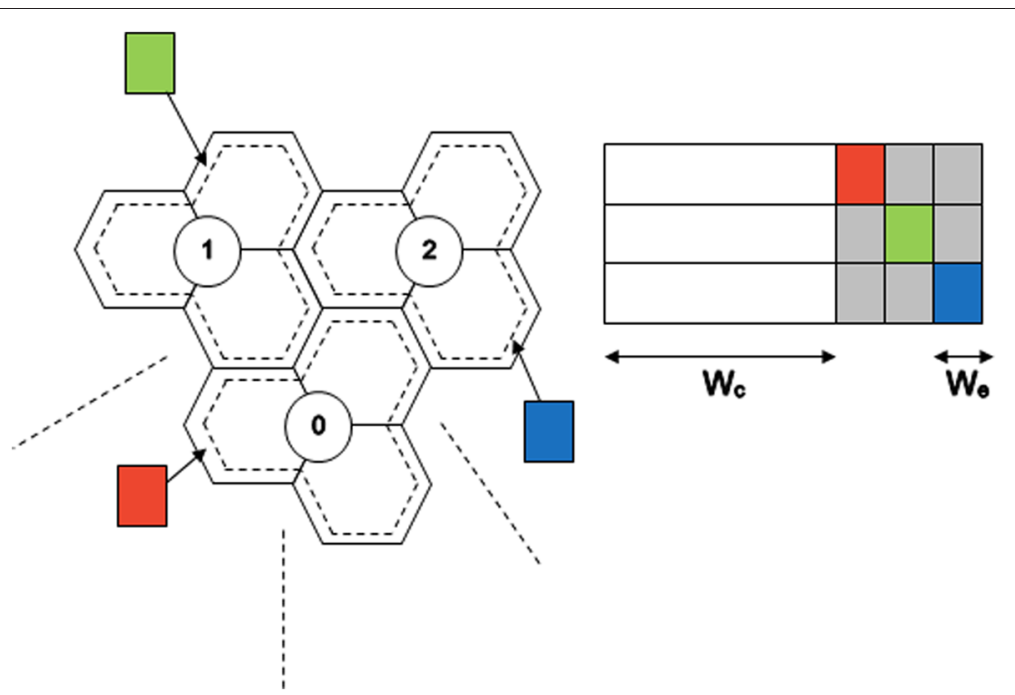

Fig. 5 Clustered multi-cell network with FFR [26] 

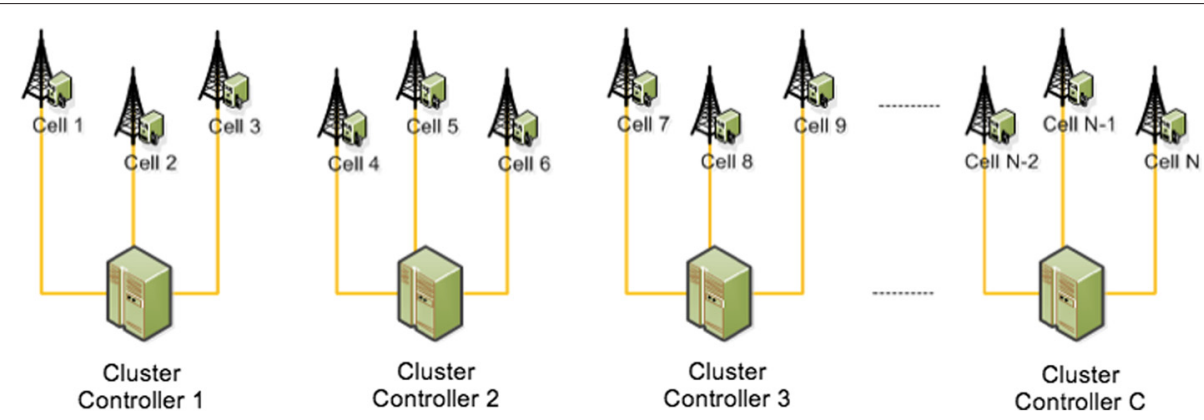

Fig. 6 CFFR distributed architecture

each BS is equipped with $N_{\mathrm{R}}=4$ antennas. The cell radius is $1 \mathrm{~km}$, and the coordination distance $D_{c}$, which is the boundary between the cluster-centre and cluster-edge, is 350 metres (i.e. 0.35R [16]). We consider shadowing and fading and the whole setup is summarised in Table 1.

Since we assume that $l_{k}=N_{\mathrm{T}}$ in this paper, the maximum number of users that can be scheduled in a cluster during each TTI for DC is 6, using (3). For CFFR, on the other hand, since cluster-centre and cluster-edge users do not interfere with each other, these two types of users can

Table 1 The setup

\begin{tabular}{ll}
\hline Cell parameters & \\
Number of cells, $N$ & 21 \\
Cluster size, $B$ & 3 \\
Cell radius, $R$ & $1 \mathrm{~km}$ \\
Coordination distance, $D_{C}$ & $350 \mathrm{~m}$ \\
User distribution & Uniform \\
Load distribution & Symmetric \\
MIMO parameters & \\
Number of transmit antennas, $N$ T & 2 \\
Number of receive antennas, $N_{R}$ & 4 \\
Channel model & \\
Carrier frequency & $800 \mathrm{MHz}$ \\
Reference distance & $100 \mathrm{~m}$ \\
Path loss exponent & 3.7 \\
Shadowing model & Log-normal \\
Shadowing variance & $10 \mathrm{~dB}$ \\
Fading & Narrowband, Rayleigh \\
Power Allocation & \\
Transmitted power of each MS & $10 \mathrm{TTls}$ \\
Scheduling & \\
Algorithm & $23 \mathrm{dBm}$ \\
Fairness time window & \\
\hline
\end{tabular}

be scheduled independently. Thus, for CFFR, the maximum number of users that can be scheduled in a cluster per TTI is 12 (i.e. six cluster-centre and six cluster-edge users). We employ the proportional fair (PF) scheduling algorithm where the time window over which fairness is imposed is set to 10 TTIs. Monte Carlo simulations are run over 100 different network realisations, and for each realisation, 1000 TTIs are considered.

\subsection{The results}

We will first study the classification accuracy of the logistic regression (LR) algorithm. This step is crucial for CFFR since the bandwidth partitions are determined accordingly. The scheduling of the users also follows this step. The classification accuracy for various loads in the cell is summarised in Table 2.

The LR algorithm performs well, and we obtain a classification accuracy between 88 and $90 \%$. The percentage of misclassified cluster-centre and cluster-edge MSs are also quantified in Table 2. If cluster-centre MSs are misclassified as cluster-edge, then this will impact the overall cell sum-rate since a lower bandwidth is allocated for them. On the other hand, if cluster-edge MSs are misclassified as cluster-edge, then this will result in raising the inter-cluster interference intensity.

In Fig. 7, we plot the cumulative distribution function (CDF) of the inter-cluster interference intensity, $\beta$. As mentioned in the setup, the users are distributed uniformly and we consider 100 such network realisations. For

Table 2 Location classification accuracy of CFFR-LR

\begin{tabular}{|c|c|c|c|c|c|c|}
\hline Load (MSs/cell) & 5 & 10 & 15 & 20 & 25 & 30 \\
\hline $\begin{array}{l}\text { Classification } \\
\text { accuracy (\%) }\end{array}$ & 88.72 & 90.3 & 87.98 & 90.62 & 90.62 & 88.94 \\
\hline $\begin{array}{l}\text { Misclassified } \\
\text { cluster-centre } \\
\text { MSs (\%) }\end{array}$ & 8.6 & 3.76 & 9.18 & 6.5 & 3.21 & 5.44 \\
\hline $\begin{array}{l}\text { Misclassified } \\
\text { cluster-edge } \\
\text { MSs (\%) }\end{array}$ & 2.68 & 5.94 & 2.84 & 2.88 & 6.17 & 5.62 \\
\hline
\end{tabular}




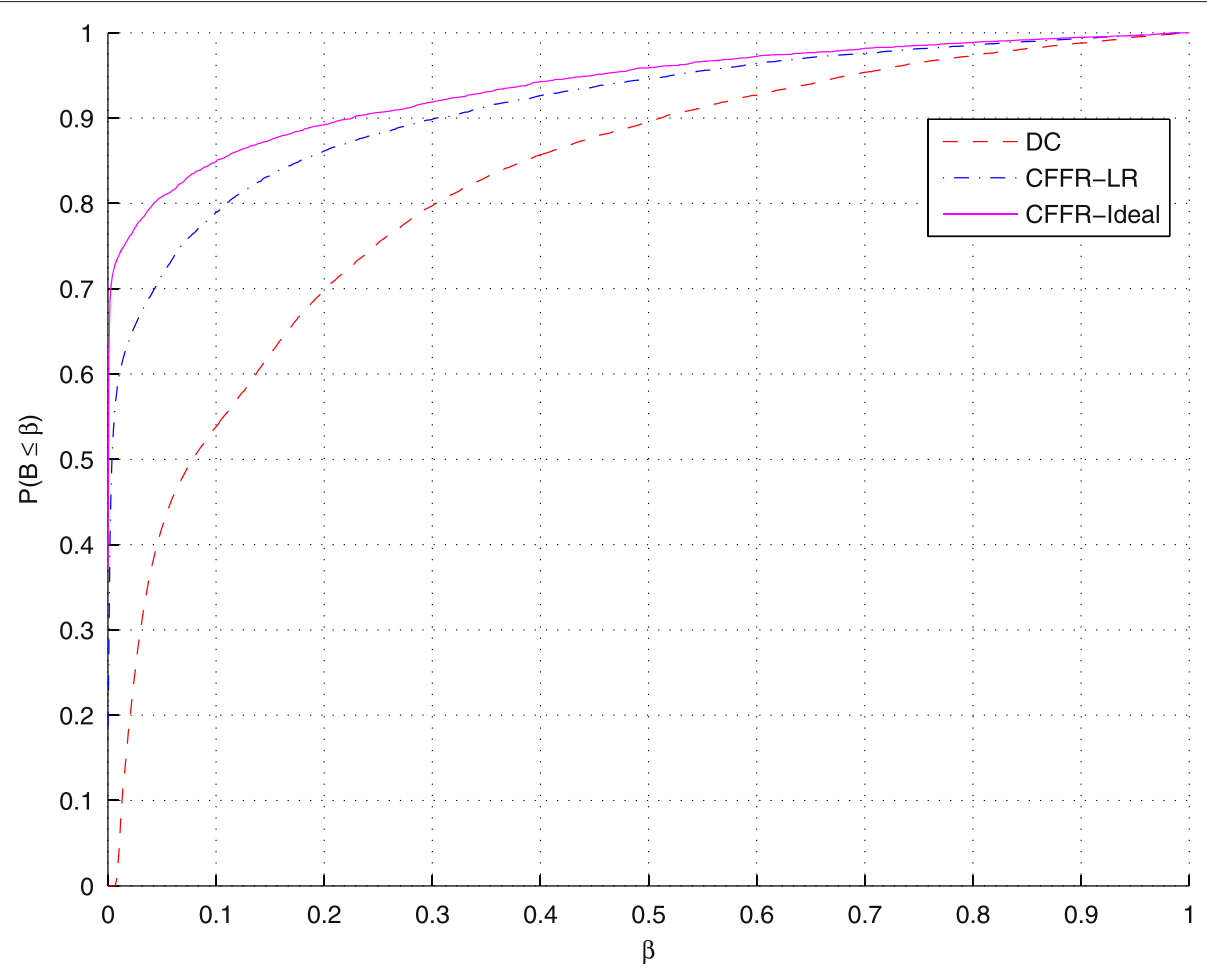

Fig. 7 CDF of the inter-cluster interference intensity

each realisation, we record $\beta$ for 1000 TTIs where users are scheduled using the PF algorithm. We can observe that DC suffers from higher inter-cluster interference than our proposed CFFR technique. For instance, the probability that $\beta$ is 0 (i.e. no interference), denoted as $\mathbb{P}(\beta=0$ ) is 0 for DC whereas it is 0.17 for CFFR-LR and 0.37 for CFFR-Ideal. It is therefore more likely that there is no inter-cluster interference for CFFR. Note that CFFR-LR suffers from more interference than its ideal counterpart due to sub-optimal location classification and the distributed architecture. The gap however is not that large. Similarly, it can be observed that $\mathbb{P}(\beta \leq 0.005)$ is 0 for DC, 0.53 for CFFR-LR and 0.72 for CFFR-Ideal. This CDF study therefore shows that CFFR is more superior than $\mathrm{DC}$ in mitigating inter-cluster interference. This is further validated by the per-cell sum rate performance shown in Fig. 8.

We can observe in Fig. 8 that the performance of DC is limited by inter-cluster interference. We have seen in the CDF of $\beta$ that $\mathbb{P}(\beta>0.005)$ is 1 , and from our theoretical study, we saw that the slightest $\beta$ is detrimental to the overall cell-rate performance (see Fig. 3 ). CFFR, on the other hand, does a better job of lowering $\beta$ and therefore shows much better sum rate performance. Since clustercentre and cluster-edge users are allocated independent sub-bands, more users can be scheduled per TTI thereby improving the overall sum rate. It can also be observed that since the SINR is lower for DC; LMMSE performs better than ZF. Since the SINR is much better for CFFR, the simpler $\mathrm{ZF}$ technique can be used for decoding. In addition, the gap between CFFR-ideal and CFFR-LR is not significant. This is because the load distribution considered in this paper is symmetric. If the load distribution was asymmetric, i.e. there are different number of users in each cell, then the distributed architecture in CFFR-LR would partition the bandwidth differently for each cluster. For instance, if one cluster had all the users in the clustercentre then it would allocate the entire bandwidth for its users. This would then increase the interference intensity to a neighbouring cluster that has users in the clusteredge. A study of the impact of asymmetric load on the performance will be done in the future.

Finally, we compare the running times of the DC and CFFR-LR algorithms in Fig. 9. For DC, we are only interested in the running time of determining the clusters and user scheduling. For CFFR-LR, on the other hand, we are only interested in the running time of performing the location classification, scheduling and bandwidth partitioning. For DC, the greedy algorithm requires computation of the joint capacity of the cluster for each iteration. If there are $K$ users in the cluster and $B$ base stations forming a cluster, the complexity of computing the joint capacity can be approximated as $O\left(K N_{T} B^{2} N_{R}^{2}\right)$. For CFFR, on the other hand, the complexity of determining 


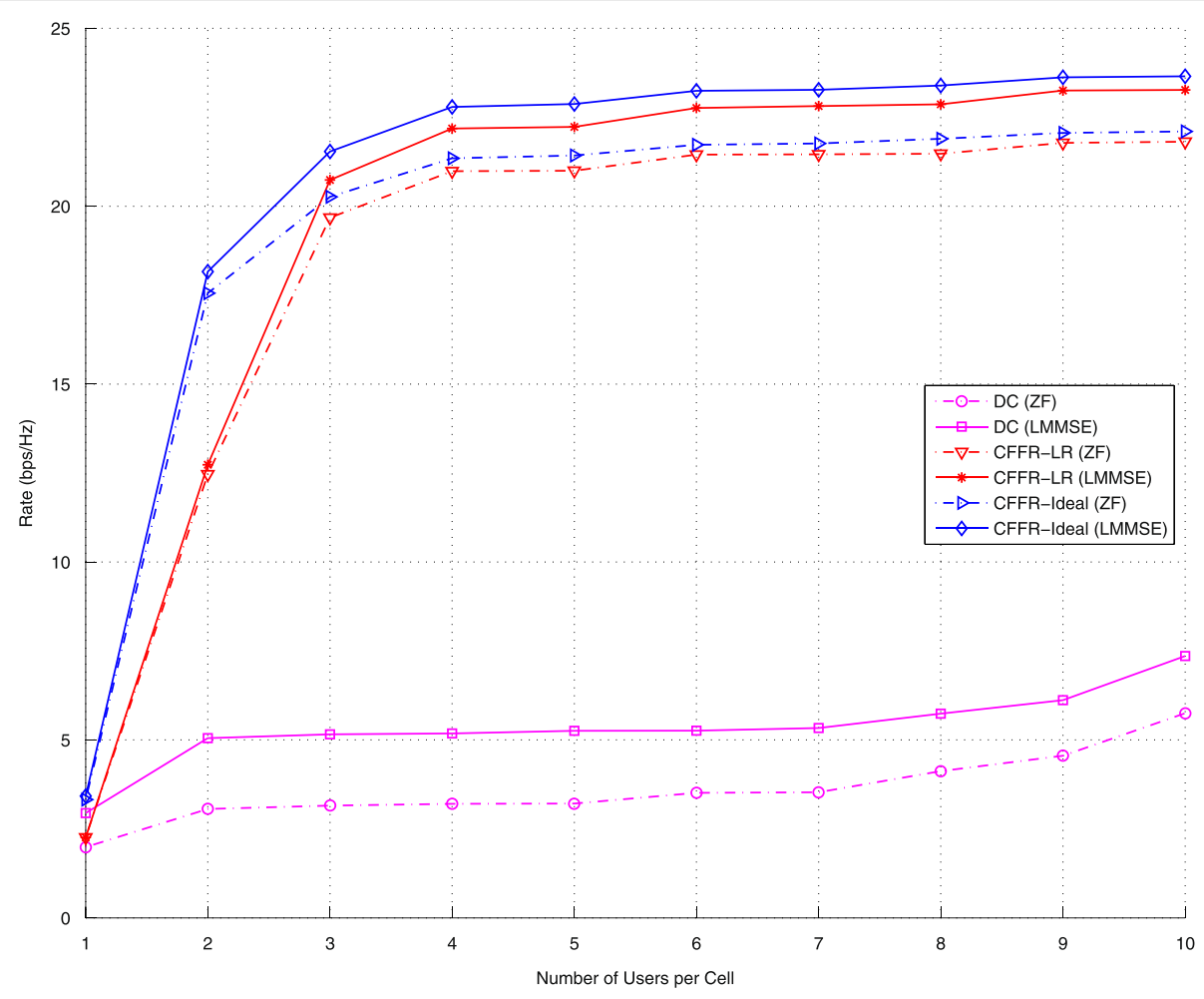

Fig. 8 Per-cell sum rate performance comparison

the user location given only two features (received power and SINR) can be approximated as $O(K)$. The complexity of user scheduling should be negligible compared to this and, moreover, should be the same for both DC and CFFR. We assume that the capacity of the backhaul is infinite and ignore the latency of CSI exchange between the BSs and the central unit. We can observe from the figure that CFFR-LR runs significantly faster than DC. In addition, we can see that the running time of DC is much more dependent on the load in the cell. We see the running time

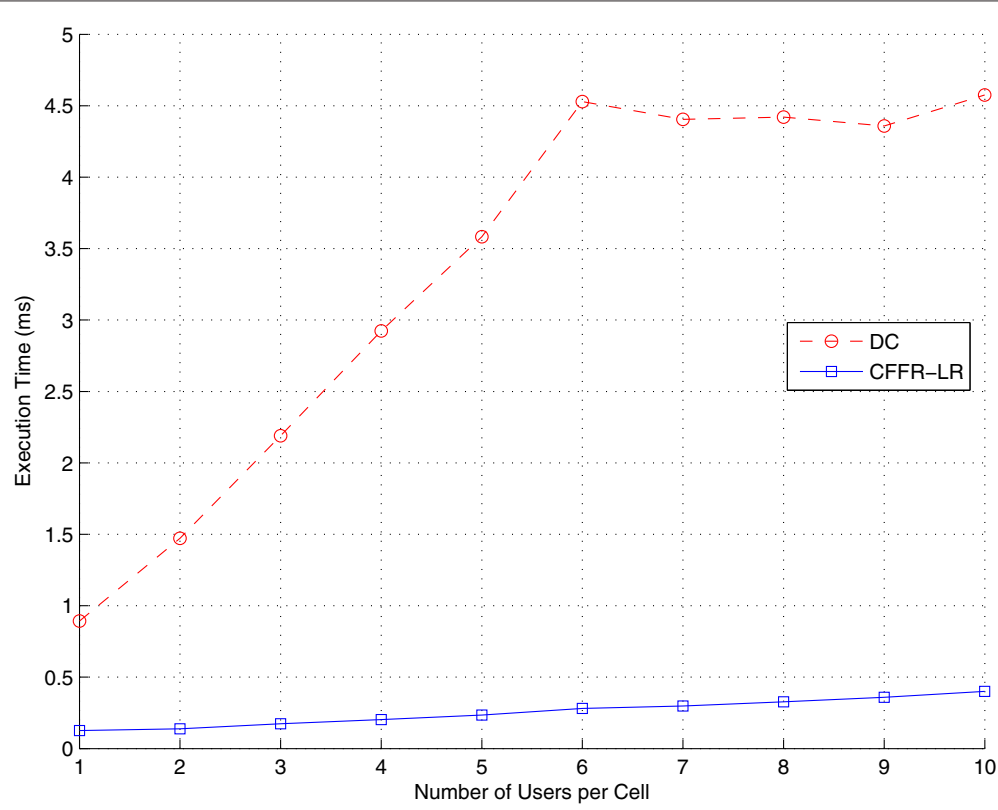

Fig. 9 Average running time comparison 
flattening after a load of 6 users per cell. This is because for DC and the setup considered, we cannot schedule more than 6 users per cell per TTI.

\section{Conclusions}

In this paper, we have proposed a practical and scalable solution to overcoming interference through static clustering and adaptive FFR. We focus on a three-cell clustered network because of its low complexity and also because we can achieve near-global-MCP performance using this cluster size. We modify the planar Wyner network and derive analytical capacity equations for a clustered network on the uplink for ZF and LMMSE decoders. We show that inter-cluster interference is the major bottleneck, and even the slightest interference intensity can have detrimental effects on the system performance. We then compare our proposed approach with the widely studied dynamic clustering technique on a more realistic network setting. For this study, we address some of the gaps in one of our earlier papers by adopting an entirely distributed architecture and by implementing a location classification algorithm based on logistic regression. We show that our proposed technique lowers the inter-cluster interference significantly when compared to dynamic clustering. As a result, the per-cell sum rate of clustered FFR is $\sim 1.5 \times$ faster especially at high loads (10 users per cell). In addition, the proposed approach is significantly less complicated to implement, requiring less computation as indicated by the run time results. The fully distributed architecture also lowers the traffic overhead on the backhaul and simplifies the implementation when compared to the hybrid and centralised architectures. In the future, we plan to extend this study to asymmetric loads and to heterogeneous networks.

\section{Competing interests}

The authors declare that they have no competing interests.

\section{Acknowledgements}

The authors would like to acknowledge Toshiba Research Europe Ltd. (TREL) and the U.K. Research Council for supporting the work done in this paper through the Dorothy Hodgkin Postgraduate Award.

\section{Author details}

${ }^{1}$ Department of Electrical and Electronic Engineering, University of Bristol, Woodland Road, Bristol, UK. ${ }^{2}$ Toshiba Research Europe, Telecommunications Lab, Bristol, UK.

Received: 2 October 2015 Accepted: 7 April 2016

Published online: 23 April 2016

\section{References}

1. G Boudreau, J Panicker, N Guo, Interference coordination and cancellation for 4G networks. IEEE Commun. Mag. 47(4), 74-81 (2009)

2. D Gesbert, S Hanly, H Huang, S Shamai Shitz, O Simeone, W Yu, Multi-cell MIMO cooperative networks: a new look at interference. IEEE J. Selected Areas Commun. 28(9), 1380-1408 (2010)
3. SW Halpern, Reuse partitioning in cellular systems. Vehicular Technology Conference, 322-327 (1983)

4. M Assaad, Optimal fractional frequency reuse (FFR) in multicellular OFDMA system. IEEE VTC Fall, 1-5 (2008)

5. Y Chang, Z Tao, J Zhang, A graph-based approach to multi-cell OFDMA downlink resource allocation. IEEE Globecom, 1-6 (2008)

6. T Novlan, JG Andrews, I Sohn, RK Ganti, A Ghosh, in IEEE Globecom. Comparison of Fractional Frequency Reuse Approaches in the OFDMA Cellular Downlink, (2010), pp. 1-5

7. Siemens, Interference mitigation - Considerations and Results on Frequency Reuse. 3GPP TSG-RAN WG1 Meeting \#42, R1-050738 (2005)

8. Huawei, Soft Frequency Reuse Scheme for UTRAN LTE. 3GPP TSG RAN WG1 Meeting \#41, R1-050507 (2005)

9. Y Liang, A Goldsmith, G Foschini, Evolution of base stations in cellular networks: denser deployment versus coordination. IEEE ICC, 4128-4132 (2008)

10. E Hossein, DI Kim, VK Bhargava (eds.), Cooperative Cellular Wireless Networks (Cambridge University Press, 2011), pp. 1-1

11. R1-093834, Backhaul Issues and its Practical Evaluation Methodology for DL CoMP. 3GPP (TSG RAN WG1 Meeting \#58) (2009)

12. O Somekh, O Simeone, Y Bar-ness, AM Haimovich, U Spagnolini, SS Shitz, An Information Theoretic View of Distributed Antenna Processing in Cellular Systems, vol. December. (CRC Press, Boston, MA, 2007)

13. O Simeone, N Levy, A Sanderovich, O Somekh, BM Zaidel, HV Poor, S Shamai, Cooperative wireless cellular systems: an information-theoretic view. Foundations Trends Commun. Inform. Theory. 8(1-2), 1-177 (2011). doi:10.1561/0100000048

14. S Venkatesan, in IEEE PIMRC. Coordinating Base Stations for Greater Uplink Spectral Efficiency in a Cellular Network, (2007), pp. 1-5

15. S Venkatesan, in IEEE PIMRC. Coordinating Base Stations for Greater Uplink Spectral Efficiency: Proportionally Fair User Rates, (2007), pp. 1-5

16. J Zhang, R Chen, JG Andrews, A Ghosh, RW Heath, Networked MIMO with clustered linear precoding. IEEE Trans. Wireless Commun. 8(4), 1910-1921 (2009)

17. S Shim, JS Kwak, RW Heath, JG Andrews, Block diagonalization for multi-user MIMO with other-cell interference. IEEE Trans. Wireless Commun. 7(7), 2671-2681 (2008)

18. A Papadogiannis, D Gesbert, E Hardouin, in IEEE ICC. A dynamic clustering approach in wireless networks with multi-cell cooperative processing, (2008), pp. 4033-4037

19. A Papadogiannis, GC Alexandropoulos, in IEEE FUZZ. The value of dynamic clustering of base stations for future wireless networks, (2010), pp. 1-6

20. P Baracca, F Boccardi, N Benvenuto, A dynamic clustering algorithm for downlink CoMP systems with multiple antenna UEs. EURASIP J. Wireless Commun. Netw. 2014(1), 1-14 (2014)

21. A Papadogiannis, H Jr Bang, D Gesbert, E Hardouin, in IEEE PIMRC. Downlink Overhead Reduction for Multi-Cell Cooperative Processing Enabled Wireless Networks, (2008), pp. 1-5

22. J-M Moon, D-H Cho, Efficient cell-clustering algorithm for inter-cluster interference mitigation in network MIMO systems. IEEE Commun. Lett. 15(3), 326-328 (2011)

23. J-M Moon, D-H Cho, in IEEE VTC Spring. Inter-Cluster Interference Management Based on Cell-Clustering in Network MIMO Systems, (2011), pp. 1-6

24. J Liu, D Wang, in IEEE WCSP. An Improved Dynamic Clustering Algorithm for Multi-User Distributed Antenna System, (2009), pp. 1-5

25. S Zhou, J Gong, Z Niu, Y Jia, P Yang, in IEEE Globecom. A decentralized Framework for Dynamic Downlink Base Station Cooperation, (2009), pp. 1-6

26. A Thampi, S Armour, Z Fan, D Kaleshi, in European Wireless. Clustered Network MIMO and Fractional Frequency Reuse for the Downlink in LTE-A Systems, (2014), pp. 1-6

27. L-C Wang, C-J Yeh, 3-cell network MIMO architectures with sectorization and fractional frequency reuse. IEEE J. Selected Areas Commun. 29(6), 1185-1199 (2011)

28. AD Wyner, Shannon-theoretic approach to a Gaussian cellular multiple-access channel. IEEE Trans. Information Theory. 40(6), 1713-1727 (1994). doi:10.1109/18.340450

29. A Grant, S Hanly, J Evans, M Ralf, Distributed Decoding for Wyner Cellular Systems. Australian Communication Theory Workshop Proceedings, 1-5 (2004) 
30. BL Ng, J Evans, S Hanly, A Grant, Information capacity of Wyner's cellular network with LMMSE receivers. IEEE ICC. 1, 583-587 (2004). doi:10.1109/ICC.2004.1312556

31. S Jing, DNC Tse, J Hou, JB Soriaga, JE Smee, R Padovani, Multi-cell downlink capacity with coordinated processing. EURASIP J. Wireless Commun. Netw. 8, 1-19 (2008). http://dl.acm.org/citation.cfm?id=1463064

32. J Xu, J Zhang, J Andrews, On the accuracy of the Wyner model in cellular networks. IEEE Trans. Wireless Commun. 10(9), 3098-3109 (2011). arXiv:1009.5900v1

33. SM Kay, Fundamentals of Statistical Signal Processing. (Prentice Hall, 1993)

34. A Thampi, S Armour, Z Fan, D Kaleshi, in IEEE International Symposium on a World of Wireless, Mobile and Multimedia Networks (WoWMoM). A Logistic Regression Approach to Location Classification in OFDMA-based FFR Systems, (2013), pp. 1-9

35. A Najjar, N Hamdi, Efficient frequency reuse scheme for multi-cell OFDMA systems. IEEE ISCC, 261-265 (2009)

36. T Novlan, R Ganti, A Ghosh, J Andrews, Analytical evaluation of fractional frequency reuse for OFDMA cellular networks. IEEE Trans. Wireless Commun. 10(12), 4294-4305 (2011)

37. Z Xu, G Li, Optimal threshold design for FFR schemes in multi-cell OFDMA networks. IEEE ICC, 1-5 (2011)

\section{Submit your manuscript to a SpringerOpen ${ }^{\circ}$ journal and benefit from:}

- Convenient online submission

$\checkmark$ Rigorous peer review

- Immediate publication on acceptance

- Open access: articles freely available online

- High visibility within the field

Retaining the copyright to your article

Submit your next manuscript at $\gg$ springeropen.com 